\title{
RESENTIMIENTO EN POLÍTICA
}

Cristóbal Astorga Sepúlveda*

RESUMEN: La noción de resentimiento puede ser analizada bajo diversas perspectivas, por de pronto la moral y la política. Se presentan algunos esquemas que permiten explicarlo y se ofrece una lectura que resalta su valor para la discusión política conectándolo con la idea de consistencia.

PALABRAS CLAVE: Argumentación racional - Igualdad - Libertad politica - Resentimiento.

ABSTRACT: The idea of resentment could be analyzed from various perspectives, for now under moral and political perspectives. Some schemes are presented that allow explaining it and a reading is offered that stresses its value for political discussion connecting it with the idea of soundness.

\section{KEYWORDS: Rational argument - Equality - Political freedom - Resentment.}

Hay cierta ambivalencia en el significado de la palabra "resentimiento". Por una parte, ella es utilizada cuando las personas sienten algún dolor o sufren los efectos de algún mal, por ejemplo, los jugadores de fútbol regularmente "resienten la lesión" o "resienten (el dolor de) la rodilla". Por otro lado, y en contextos distintos, tener resentimiento involucra tener ciertas actitudes hacia algunas personas, por ejemplo, se dice que alguien "está lleno de resentimiento" y se señala una cierta actitud que el aludido ha tenido con respecto a otro, sea de forma directa o indirecta. Sin embargo, no se dice del futbolista que sea un resentido, como sí ocurre en el segundo caso. El resentimiento del futbolista no sería sino otra cosa más que les pasa a las personas dada su constitución física esencialmente vulnerable, en cambio, el resentimiento en el segundo caso sería una forma particular de ser que tienen las personas (aunque, ¿todas las personas?). Si habláramos del resentimiento en el primer caso no diríamos, salvo que el jugador fuera un quejoso, que se trata de algo malo o molesto; en el segundo, sin embargo, sí (aunque, ¿todos diríamos lo mismo?).

En la primera parte examino algunas estrategias argumentativas que pueden explicar el resentimiento, ya sea por referencia a un esquema de virtudes, de dominación o de genealogía de las ideas morales. Propongo en la segunda parte, una explicación distinta que discute el valor epistémico del resentimiento; en este esquema será necesario decir algo acerca del trabajo argumentativo realizado sobre el resentimiento y su pertinencia en la esfera pública.

\footnotetext{
* Alumno egresado, y ayudante ad honorem de los cursos Filosofía (de la) Moral e Historia de la Filosofía del Derecho, Facultad de Derecho, Universidad de Chile. Agradezco los comentarios hechos a versiones preliminares por parte de Marcos Andrade Moreno, Marianne González Le Saux, M. E. Orellana Benado, Édison Orellana Ramos, Esteban Pereira Fredes, Ernesto Riffo Elgueta y Francisco Vergara Letelier. Esta comunicación fue preparada en el marco del proyecto de investigación "Derecho y Filosofía Moral" (DI SOC 06/21-2). Dirigir comentarios derechamente a kastorgas@yahoo.es.
} 


\section{Primera parte.}

Consideremos algunos juicios:

(1) "No aceptó nuestra invitación a comer. Es un resentido".

(2) "Él representa todo el resentimiento de cierto sector de la sociedad".

(3) "¿Obtuvo la beca? Debe haber sido con todo su esfuerzo".

(4) "Nadie que viva tan cerca de la cordillera puede decirse de izquierda".

Los dos primeros atribuyen a un agente cierta característica, ser resentido o ser un representante de él, aunque en el caso número (1) claramente puede ilustrar un rango de situaciones más amplio (como si el invitado estuviera sentido por alguna situación anterior, digamos su cumpleaños fue olvidado). Los dos últimos ilustran actitudes que bien podrían gatillar (2): en (3) hay un cierto escepticismo acerca del mérito del beneficio obtenido, lo que se expresaría irónicamente con alguna inflexión en la palabra resaltada; en el caso número (4), en cambio, puede no haber un tono irónico ni una cierta indiferencia, sino que puede $-\mathrm{y}$ regularmente así será- ir acompañado de alguna forma de indignación; ambos se caracterizan, al parecer, porque involucran un juicio de valor distinto al expresado por el caso número (1) y número (2). Es de notar, también, que los juicios impares presentan alguna referencia oblicua a un rango de actitudes no especificado, mientras los juicios pares se centran directamente en dichos, y no tanto a hechos (si puede ser sostenida una distinción tan tajante).

Creo que hasta aquí podrían examinarse algunas estrategias explicativas. Podría decirse que el "resentimiento" es un sentimiento legítimo si se refiere a un dolor o daño provocado por una acción voluntaria. ${ }^{1}$ En cambio, cuando lo único que hace es enmascarar algún otro tipo de sentimiento o vicio (por ejemplo, envidia), es digno de ser enrostrado y combatido (aunque, chacemos eso con la envidia?). Lo primero puede ser correcto, pero nos deja con el problema de tomar un desvío para entender qué expresan los dos últimos juicios. La envidia puede jugar algún papel en el caso número (3), pero es más dudoso -sin caer en una explicación reduccionista- si lo hace en el caso número (4). Para Aristóteles, la envidia puede indicar un tipo de autorreproche, y por ello es una forma de tristeza: "También [envidiamos] a aquellos cuyas ganancias o éxitos son un reproche para nosotros, (...) pues es evidente que no hemos conseguido el bien que ellos han logrado.”2 Pero ¿qué se reprocharía el resentido señalado en el número (4)? ¿Qué clase de "bien perseguido" está en juego?

La envidia como explicación del resentimiento es ciertamente la salida más fácil. Principalmente porque permite identificar un objeto, aquello envidiado, como algo bueno; de ahí que la acción del envidioso no se dirija necesariamente a destruir al envidiado, sino a obtener el bien que él detenta. Pero esto hace de la envidia algo semejante a la codicia. La envidia no puede consistir solamente en un sentimiento acerca de objetos externos. El aspecto moral de la envidia reside en el carácter relacional que vincula al envidiado y al envidioso, y donde importan los atributos del primero (y esto sugiere que la envidia requiere de algún tipo de cercanía). El dolor que experimenta el envidioso se debe a la carencia de algo y su deseo de

\footnotetext{
${ }^{1}$ Esta es una forma de analizar el resentimiento en filosofía moral; véanse STRAwSON, P. "Libertad y resentimiento”, en su Libertad y resentimiento (trad. J. J. Acero). Buenos Aires, Paidós, 1996. y WALlace, J. "Ressentiment, Value, and Self-Vindication, Making Sense of Nietzsche's Slave Revolt", en su Normativity and the Will. Oxford: Oxford University Press, 2007.

${ }^{2}$ Aristóteles, Retórica, $1388 \mathrm{a} 15$.
} 
obtenerlo, pero no porque se trate de algo valioso en sí mismo, sino porque es poseído por el envidiado y no por cualquier otro. En muchos casos el envidioso se compara con el envidiado, quien puede llegar a asumir el carácter de un rival; en esa comparación el primero se observa degradado y anhela corregir la asimetría.

Si este fuera el caso del resentimiento, tendríamos que explicarlo diciendo que el resentido se percibe dañado. Su dolor no emana de la distancia que lo separa de la cordillera, sino de que otros florezcan cuando él no lo hace. Por ello, y bajo este esquema, (4) no debiera equipararse a un mecanismo compensatorio (o preferencia adaptativa al estilo de la fábula del zorro y las uvas), sino más bien al comienzo de una estratagema dirigida o bien a socavar el florecimiento ajeno o bien a posibilitar el propio.

No estoy seguro que cuando se descarta el resentimiento reduciéndolo a la envidia se quiera decir todo esto, y aquello por dos razones: en primer lugar, la envidia es percibida como un vicio, como algo malo, una mezquindad: en lugar de alegrarme por otros (cuando obtienen algo), hago un comentario mezquino, como en (3). Así, el resentimiento se torna algo superficial, vinculado principalmente a bienes que no dicen relación con algún sujeto. En segundo lugar, la banalización del resentimiento involucra su esterilidad: nada bueno nace de él, al contrario, amarga la vida y sólo contribuye a adoptar una posición negativa frente al mundo. El resentimiento es percibido como enfado, rabia, desprecio. ${ }^{3}$

Una segunda estrategia explicativa, diría que el resentimiento es una actitud dirigida desde un sector social hacia otro. No se mueve bidireccionalmente, sino que sólo algunos son denominados resentidos por otros. Eso expresa el caso número (2), y se arroga, por de pronto, el uso del término; no tendría mucho sentido decir:

(5) “No lo contrató porque era moreno, iqué resentido!”, cuando regularmente a ese tipo de personas se las califica como "discriminadoras" o "clasistas". Este análisis tiene sus ventajas, ilustra, sin tener que recurrir al vocabulario moral de la envidia, el tipo de conflicto involucrado: la dominación y explotación de una clase por otra.

La idea de dominación ha recibido desde hace tiempo un resurgimiento a partir de la obra de Philip Pettit, principalmente en su Republicanismo, quien ha vinculado su entendimiento de la libertad política a la idea de no-dominación. ${ }^{4}$ Resalto dos puntos del análisis de Pettit: la dominación es un fenómeno normativo, es decir, depende de ciertas reglas que organizan la vida en comunidad por ejemplo, las reglas que establecen las relaciones de subordinación y obediencia y que establecen ciertas atribuciones para algunos y no para otros. En segundo lugar, la extensión y la intensidad de la dominación son cuestiones diferentes que comprometen la libertad; la libertad como no-dominación puede ser aumentada si se reducen ambas, o bien si se elaboran mecanismos por los cuales el ámbito de acciones no-dominadas es incrementado o transformado (a lo que Pettit se refiere como "el modo expansivo de incrementar el alcance de la libertad"), es decir, si se reduce aquello que condiciona la libertad. ${ }^{5}$

Luego, si afirmáramos todo aquello es decir, si el resentimiento representa la actitud que adquiere una clase dominada frente a una clase dominante, esto puede suponer que la

${ }^{3}$ Todavía podrían compararse la envidia y los celos; véase BEN-ZE’Ev, A. "Envy and jealousy", Canadian Journal of Philosophy, (20), 1990.

${ }^{4}$ Véase también PETTIT, P. "Keeping Republican Freedom Simple: On a Difference with Quentin Skinner", Political Theory, (30), 2002. Para algunas críticas, LARmOre, C. “A Critique of Philip Pettit's Republicanism”, Philosophical Issues, 11, 2001.

5PETTiT, P. Republicanismo. Una teoría sobre la libertad y el gobierno (trad. T. Domènech). Barcelona, Paidós, 1999, pp. 105-109. 
dominación posee niveles altos de extensión e intensidad, pero también que no existe la posibilidad de operar una transformación en el ámbito de acciones no-dominadas disponibles. En pocas palabras, que el resentimiento no es tan solo una queja acerca de las reglas que establecen dominación, sino un reclamo acerca del carácter baldío de la libertad disponible. El resentimiento pareciera expresar que esa libertad emana de una visión de la política paternalista, que otorga algo que llama "libertad", pero que en realidad no se asemeja a ella.

La idea más predominante, a la luz de lo anterior, es que el resentimiento emana de ciertos sectores sociales, los peores situados, que elevan reclamos por el carácter que tiene su libertad. Pero también encierra un reclamo acerca de cómo se configura la libertad. La objeción maquiavelizante -la idea repetida hasta el cansancio que los políticos han renunciado a la salvación de sus almas- no viene del todo al caso aquí, ya que los reclamos no son dirigidos de ordinario solamente contra los políticos. La idea de que podemos reclamar legítimamente integridad de los demás ciudadanos en cuanto concurren a la esfera pública, es inconfundible con la idea de responsabilidad política exigible a los representantes políticos. ${ }^{6} \mathrm{Si}$ la noción de responsabilidad política puede parecer escurridiza, entonces más debiera parecerlo la idea de integridad ciudadana (un término que parece salido de algún informe del Comité de Salubridad Pública). Espero volver a remitirme acerca de ello más adelante.

Queda todavía una tercera explicación, la nietzscheana. El argumento es conocido, pero no está de más presentarlo brevemente, en particular porque se trata de un uso explícito del término. En el primer tratado de La genealogía de la moral, Nietzsche avanza su tesis acerca de la rebelión de los esclavos. Según ella, los esclavos habrían elaborado un sistema nuevo de valores que desmanteló los viejos; así, los valores aristocráticos de "bueno y malo" fueron reemplazados por los valores cristianos de "bueno y malvado". Ese proceso fue posible por aquello que Nietzsche identifica con resentimiento. Permítanme citar con largueza el inicio de la sección 10:

"La rebelión de los esclavos en la moral comienza cuando el resentimiento mismo se vuelve creador y engendra valores: el resentimiento de aquellos seres a quienes les está vedada la auténtica reacción, la reacción de la acción, y que se desquitan únicamente con una venganza imaginaria. (...) Esta inversión de la mirada que establece valores - este necesario dirigirse hacia fuera en lugar de volverse hacia sí - forma parte precisamente del resentimiento: para surgir, la moral de los esclavos necesita siempre primero de un mundo opuesto y externo, necesita, hablando fisiológicamente, de estímulos exteriores para poder en absoluto actuar, - su acción es, de raíz, reacción". ${ }^{7}$

"Resentimiento" describe un afecto negativo dirigido hacia los nobles. Los esclavos se dan cuenta que existe una deprivación estructural, que los pone en una situación comparativamente menoscabada. El mecanismo psicológico del resentimiento, es la expresión de la orientación emocional negativa dirigida hacia los poderosos, y en ese sentido no funciona

${ }^{6} \mathrm{El}$ problema más conocido es el llamado de las "manos sucias"; véase Walzer, M. "Political Action: The Problem of Dirty Hands", Philosophy and Public Affairs, (2), 1973. Michael Stocker resalta el sentido de pérdida y los sentimientos involucrados en la ejecución de una acción que ensucia; STOCKer, M. Plural and Conflicting Values. Oxford, Clarendon Press, 1990, pp. 9-36.

${ }^{7}$ NieTzSCHE, F. La genealogía de la moral (trad. A. Sánchez Pascual), -2005, 6ª reimp.-, Madrid, Alianza, 1997, p. 50. 
según la lógica de la racionalidad estratégica (que busca medios para alcanzar fines), sino más primitivamente como un patrón de auto-interpretación emocional, permitiéndoles así hacer sentido de su experiencia del mundo, en la cual se perciben desmedrados. ${ }^{8}$

El argumento de Nietzsche no es precisamente político. Ofrece en cambio, una explicación psicológica de la percepción que un grupo tiene de su interacción con otro, y cómo puede engendrarse de ahí algo nuevo; se trata también de un diagnóstico del estado patológico en que se haya la moralidad moderna. No estoy seguro cómo podría equipararse ello a los efectos de una revolución política, sin que además tengamos que vernos forzados a entender por revolución un movimiento particularmente abarcante (pero puede ser que yo tenga una concepción demasiado razonable o demasiado ingenua de lo que es una revolución). Con todo, e incluso descartando la cuestión política del argumento de Nietzsche, es patente que el resentimiento apunta a una forma de exigir responsabilidad, de culpar a otro, y también, es obvio, de castigarlo. Esto puede jugar algún rol en instancias pre-políticas que contribuyen a dar forma a nuestras instituciones propiamente políticas, pero es un punto que escapa a las preocupaciones de estas páginas. ${ }^{9}$

\section{Segunda parte.}

Hasta aquí me he ocupado de ofrecer tres posibles explicaciones del resentimiento y su vinculación con la política. Antes de sugerir otra, quisiera retomar algunas cuestiones que han quedado pendientes.

En primer lugar, el resentimiento es un sentimiento gatillado por diversas actitudes, regularmente aquellas que nos provocan daño. Tratándose de maltrato, el resentimiento aflora como un tipo de actitud reactiva justificada, en ese caso, el resentimiento es legítimo porque con ello reconocemos la libertad en la acción del otro y lo tratamos como un igual. Siempre queda el problema de determinar cuándo estamos frente a un maltrato y ello no es un problema menor tratándose del resentimiento en política. Por lo mismo, ese resentimiento será asemejado a un rango de actitudes que van desde la indignación hasta la cólera.

Distingo ese rango porque no parece obvio en qué extremo se ubica el resentimiento. El esquema de las virtudes y los vicios, valiéndose de la envidia, debería asignarlo con cercanía a la cólera, o si se prefiere, el resentimiento resulta de la combinación de envidia y cólera. Esto es un problema, no sólo para los éticos de las virtudes, que reprobarían con seguridad semejante cóctel de vicios, sino también para quienes exigen que en el debate público los individuos concurran con razones y no con pasiones. Y esto porque ellas nos nublan y hacen que los individuos no se muestren con sus intereses reales. Es la autenticidad, muchas veces,

\footnotetext{
${ }^{8}$ Me baso en Wallace, J. “Ressentiment, Value, and Self-Vindication: Making Sense of Nietzsche's Slave Revolt”, en su Normativity and the Will. Oxford, Oxford University Press, 2006. La "interpretación expresiva" de Wallace se opone a la "interpretación estratégica", como él mismo las llama, en tres sentidos: no interpreta el resentimiento como el mero deseo de infligir daño o sufrimiento a otro; no oscurece el carácter histórico de la propuesta de Nietzsche; no hace de la revuelta de los esclavos un proyecto que se autodestruye (en el sentido de que la nueva tabla de valores exigiría, una vez que la revuelta triunfara, cambiar la actitud reactiva que los esclavos dirigían hacia los nobles). Véase también ibid., pp. 218-9 para una comparación con la envidia. ${ }_{9}^{9}$ WALlaCE, J. op. cit. (n. 8), pp. 72-4.
} 
una exigencia de la deliberación política si se quiere que ella sea algo más que razonamiento estratégico. ${ }^{10}$

En segundo lugar, si el resentimiento pudiera ser conceptuado como un reclamo hecho por un cierto sector de la sociedad hacia otro, podemos preguntar si no se trata tan sólo de una forma retórica de presentar un argumento en favor de la igualdad. El reclamo suele ser interpretado como uno acerca de la igualdad como horizonte o meta política, y para decirlo con los conservadores, un reclamo de igualdad absoluta termina siendo la antesala de la igualdad rapaz. Pero no creo que en eso consista precisamente el reclamo, me parece que su pretensión es más modesta: reclama acerca de la suficiencia de la distribución. La idea central parece ser no que otros tengan tanto más, sino que tienen tanto más porque otros tienen tanto menos, o que tienen tanto más cuando otros no tienen lo suficiente. Esa es la diferencia entre un argumento en favor de la igualdad y otro en favor de la suficiencia. ${ }^{11}$ Siempre es más fácil leer todo eso, como codicia del bien ajeno.

En tercer lugar, la creatividad. Las condiciones en las cuales se crea algo no siempre mantienen una relación de homogeneidad con lo creado. Es lo que llama la atención cuando nos enteramos que durante el régimen nazi florecieron expresiones musicales tan distintas como el decadente aristocraticismo del Dr. Strauss y la Entartete Musik (la "música degenerada", y por lo mismo prohibida), que por su parte albergaba tanto al romanticismo desbordado de Schreker como a la parodia mundana de Ullmann. Es fácil, y no demasiado contraintuitivo, realizar así una interpretación positiva del resentimiento: el lugar común que el odio nada engendra, solo el amor es fecundo, es más bien el porqué las canciones de pastoral son un género tan insípido.

Por supuesto que esas condiciones no equivalen a la motivación ni a la intención del agente. Pero incluso si no se es tan fino en las distinciones, es posible percibir que el argumento de Nietzsche no es una lectura ni optimista ni pesimista del resentimiento, sino tan solo el retrato de la psicología moral de un grupo específico, los esclavos. Que el resentimiento se vuelva creador no indica tampoco que la agencia involucrada sea autónoma o libre, ya que de alguna manera responde a una estructura teleológica a la que Nietzsche parece oponerse. $^{12}$

Hechas estas prevenciones, es posible decir algo más acerca del resentimiento. Un punto no explorado con el suficiente interés por las anteriores explicaciones es la idea de consistencia. ${ }^{13}$ En su ensayo acerca de la cólera, Montaigne ejecuta un ligero discurso a propósito de la distancia entre el dicho y el hecho:

"Hay sin duda bella armonía cuando el decir y el hacer van unidos, y no quiero negar que sea el decir de mayor autoridad y eficacia, cuando está respaldado por las obras. Como decía Eudámidas oyendo a un filósofo discurrir sobre la guerra: Son

\footnotetext{
${ }^{10}$ Cohen, J. "Deliberation and Democratic Legitimacy", en A. Hamlin y P. Pettit, eds., The Good Polity. Oxford: Blackwell, 1989. Para la autenticidad como ideal ético, AppiAH, K. La ética de la identidad (trad. L. Mosconi). Buenos Aires, Katz, 2007, pp. 47-54, $168-175$.

${ }^{11}$ Tomo la distinción de FrAnkfurT, F . "La igualdad como ideal moral", en su La importancia de lo que nos preocupa (trad. V. I. Weinstabl y S. M. de Hagen). Buenos Aires, Katz, 2005.

${ }^{12}$ No es un punto sobre el cual podría detenerme demasiado; me remito para ello a VILLA, D. "Beyond Good and Evil: Arendt, Nietzsche, and the Aestheticization of Political Action", Political Theory, 20, 1992, esp. pp. 277-87.

${ }^{13}$ No explorado por las anteriores explicaciones, pero sí por CoHEN, G. A. If You're an Egalitarian, How Come You're So Rich? Cambridge, Massachusetts \& London: Harvard University Press, 2000, en esp. pp. 148-79.
} 
bellas estas palabras, mas no es creíble el que las dice, pues no tiene los oídos acostumbrados al son de las trompetas". ${ }^{14}$

"Jamás leo a autor alguno -concluye el párrafo Montaigne-, ni siquiera a aquellos que tratan la virtud y los deberes, sin investigar cuidadosamente cómo fue." La cautela de Montaigne lo dirige a indagar en la constitución moral de los autores para cerciorarse de la correspondencia entre aquello que dicen y aquello que hacen.

Hay cierta semejanza entre el ejemplo de Eudámidas y el argumento de autoridad (ad verecundiam), y hay también cierta conexión entre la cautela de Montaigne y el argumento satanizante (ad hominem). Ambas clases de argumentos toman como eje a otra persona que ha emitido un juicio o comentario, el primero respaldando lo dicho por esa persona por referencia a la particular investidura que ella ostenta, el segundo objetándolo dada la calidad de la persona que lo ha emitido. El ejemplo de Eudámidas sería un caso de argumento de autoridad al revés, es decir, uno en el cual se rechaza lo dicho por no ostentar la persona la autoridad requerida. Nótese así que los argumentos que hacen referencia a una autoridad no reposan de forma absoluta en ella, sino que tanto en cuanto los dichos tengan relación con el rango de conocimiento que la autoridad podría manejar dada su especialidad, una forma de división cognitiva del trabajo. Decir, entonces, que es cierto todo lo que la autoridad diga sería un argumento ad hominem al revés, un argumento que respalda lo dicho por ser la persona quien es, al margen de su ámbito de competencia autoritativa. ${ }^{15}$

Pero es de notar que la reserva de Montaigne no opera en el mismo nivel que un argumento satanizante, $y$ es en ese mismo sentido en el cual el resentimiento cobra valor epistémico. La imagen que ofrece es ante todo una advertencia acerca de lo que otros están diciendo, principalmente mediante la indicación de una suspensión de la credulidad: los argumentos desplegados en el espacio público blanden razones, pero podemos mostrarnos escépticos acerca de cuán compatibles terminan siendo con su emisor.

Un caso en el cual podemos desconfiar de la razón es el de la racionalización. ${ }^{16}$ Se trata de argumentos cuyo razonamiento es irreprochable desde un punto de vista formal, pero cuya motivación dista de ser la persecución de la verdad. Esto puede observarse por la exhibición de datos selectivos, objeciones a posturas contrarias extremadamente elaboradas y reticencia a aceptar las propias objeciones como auto-refutativas. En el caso de la racionalización la motivación, muchas veces es reconducible a prejuicios o a compromisos irrestrictos con determinadas posturas teóricas. Sin embargo, la cautela con la que podemos enfrentar esos argumentos no siempre tomará la forma de cuestionar la compatibilidad del argumento con la conducta del emisor, y ello debido al alto grado de abstracción que puede tomar el primero.

Pero incluso cuando sea así, cuando en política o en filosofía política presenciemos sutileza y complicación, es lícito preguntarse qué relación guardan las cadenas argumentales

\footnotetext{
${ }^{14}$ Montaigne, M. “De la cólera”, en sus Ensayos, vol. II (trad. A. Montojo). Madrid, Cátedra, 2005, p. 452.

${ }^{15}$ Pero esto es así tan solo por la caracterización que he hecho del argumento ad bominem. Hay quienes lo presentan como el argumento por el cual se hacen objeciones a una persona oponiéndole sus propios argumentos o actos previamente realizados. Las versiones invertidas del argumento de autoridad también podrían ser tratadas de otras formas, dependiendo de la cláusula que se niegue: podría respaldarse un argumento por carecer la persona de autoridad o podría rechazárselo por tenerla, y parecen formas del tipo "suerte del principiante" y "no ves el bosque por ver los árboles" respectivamente.

${ }^{16}$ Kornblith, H. "Distrusting Reason", Midwest Studies in Philosophy, (23), 1999. Agradezco a Ernesto Riffo la referencia.
} 
con la conducta de quien las emite. Planteo el asunto de esta forma para evitar colapsar, en aras de una teoría de las emisiones realizativas, la distinción entre hacer y decir. La idea de hacer cosas con palabras en política es llamativa, pero regularmente no es más que la salida de emergencia más cercana cuando se quiere evitar el derrumbe de un argumento. También me parece que ese colapso impide enfrentar una objeción intuitivamente fuerte: que una cosa es decir algo en público, y otra distinta lo que cada cual hace en su vida privada. Esa objeción lleva mucha razón, pero es dudoso que su ventaja sea en un terreno común.

Una cosa es sostener que el ámbito privado debe ser conceptuado como secreto y otra distinta que deba serlo como discreción. Muchas de las decisiones personales que los sujetos toman demandan ser tratadas como casos de privacidad discrecional, es decir, casos donde existe cierta soberanía para decidir; pero de ahí no se sigue que esas mismas decisiones puedan ser cubiertas por la noción de privacidad como secreto. Siempre es posible exigir que las decisiones personales carezcan de intromisión externa, pero ello no impide que sean conocidas por terceros. Del conocimiento a la evaluación hay un paso discreto, pero sería necesario establecer con respecto a qué se hace la evaluación, que es justamente el punto donde podemos preguntarnos si el criterio de "lo dicho en público" puede servirnos de algo. Sin embargo, no es precisamente la comparación entre "lo público" y "lo privado" un tipo de evaluación, sino más bien una forma de constatar cierta consistencia. Ahora bien, si la consistencia fuera un valor, digamos que las personas consistentes son admirables, entonces la evaluación se presenta de todas formas, solo que por rebote. Quizá erigir la consistencia como un valor ciudadano sea elevar una exigencia de virtuosismo algo opresiva; y por ello, me parece que el resentimiento hace un mejor trabajo cuando sólo funciona como advertencia y no como tribunal de la conciencia. ${ }^{17}$

Puede también objetarse a la sospecha del resentido que su argumento no está respaldado por razones. Creo que el tiro no da en el blanco: la idea de elevar una pretensión de suspensión de la credulidad no es precisamente un argumento, sino tan solo un caveat, una advertencia acerca de la clase de argumento que se está presentando, más todavía cuando esos argumentos tienen alguna incidencia en la forma en que las comunidades organizan su vida cooperativa. Esto puede exigir, en el marco de alguna concepción política específica, una ciudadanía desconfiada y alerta, como alguna variedad de republicanismo ha sugerido, pero no se trata de una práctica exclusivamente ligada a esa teoría.

Subyace a esta objeción la preocupación por delimitar el rango de argumentos que podemos sensatamente aceptar en el ámbito público. Esto es ciertamente una cuestión persistente en teoría política, cuya razón es más bien negativa: se busca excluir. La idea que la inclusión de ciertos argumentos permite, como una bola de nieve rodando cuesta abajo, que a la larga todo valga ilustra un temor razonable, aunque muchas veces excesivamente celoso al momento de implementar la prueba de admisión: eliminando las exigencias estrepitosas y los gritos iracundos, eliminamos también las lágrimas y la risa. ${ }^{18}$ Es cierto que muchas veces el

\footnotetext{
${ }^{17}$ Cohen, G. A. op. cit. (n. 13), pp. 167-8, también se hace cargo del argumento del "espacio privado", pero su tratamiento es más sustantivo, discutiendo la forma que debería tener.

${ }^{18}$ JONSON, J. "Argumentos en favor de la deliberación. Algunas consideraciones escépticas”, en J. Elster, comp., La democracia deliberativa (trad. J. M. Lebrón). Barcelona: Gedisa, 2001, pp. 207-33. Véanse también los reparos hechos a las concepciones deliberativas y dialógicas en ELSTER, J. "The Market and the Forum: Three Varieties of Political Theory", en J. Elster y A. Aanund, eds., The Foundations of Social Choice Theory. Cambridge, Cambridge University Press, 1986. y BASU, S. "Dialogic Ethics and the Virtue of Humor", The Journal of Political Philosophy, 7, 1999.
} 
resentimiento puede ser solo un gesto de ofuscación, algo así como que ninguna forma de vida debiera florecer tan cerca de la cordillera, pero incluso en ese caso puede servir para llamar nuestra atención. Que hayan flores exóticas en la nieve es parte de su exotismo, pero quizá por lo mismo debiéramos preocuparnos de tratarlas con pinzas.

\section{REFERENCIAS BIBLIOGRAFICAS.}

ApPIAH, Kwame Anthony. La ética de la identidad (trad. L. Mosconi). Buenos Aires, Katz, 2007.

BASU, Sammy. "Dialogic Ethics and the Virtue of Humor", The Journal of Political Philosophy, (7), 1999.

BEN-ZE'Ev, Aaron. "Envy and jealousy”, Canadian Journal of Philosophy, (20), 1990.

Bohman, James y William Rehg, eds. Deliberative Democracy. Essays on Reason and Politics. Cambridge, Massachusetts \& London, MIT Press, 1997.

CoHen, G. A. If You're an Egalitarian, How Come You're So Rich? Cambridge, Massachusetts \& London: Harvard University Press, 2000.

CoHEN, Joshua . "Deliberation and Democratic Legitimacy", en A. HAMLIn y P., 1989.

PETTIT, eds., The Good Polity. Oxford, Blackwell. También en BOHMAN y REHG, 1997.

ELSTER, Jon. "The Market and the Forum: Three Varieties of Political Theory", en J.

Elster y A. AAnund, eds., The Foundations of Social Choice Theory. Cambridge: Cambridge University Press. También en BOHMAN y REHG, 1997.

FRANKFURT, Harry. "La igualdad como ideal moral", en su La importancia de lo que nos preocupa (trad. V. I. Weinstabl y S. M. de Hagen). Buenos Aires, Katz, 2005

JOHNSON, James. "Argumentos en favor de la deliberación. Algunas consideraciones escépticas”, en J. Elster, comp., La democracia deliberativa (trad. J. M. Lebrón). Barcelona, Gedisa, 2001.

Kornblith, Hilary. "Distrusting Reason", Midwest Studies in Philosophy, 23, 1999.

LARMORE, Charles. “A Critique of Philip Pettit's Republicanism”, Philosophical Issues, 11, 2001. 
Montaigne, Michel de. "De la cólera", en sus Ensayos, vol. II (trad. A. Montojo). Madrid, Cátedra, 2005.

NiETzSCHE, Friedrich. La genealogía de la moral (trad. A. Sánchez Pascual). Madrid, Alianza, 1997 [2005, 6 reimp.].

PETTIT, Philip. Republicanismo. Una teoría sobre la libertad y el gobierno (trad. T. Domènech). Barcelona, Paidós, 1999.

. "Keeping Republican Freedom Simple: On a Difference with Quentin Skinner", Political Theory, 30, 2002.

STOCKER, Michael. Plural and Conflicting V alues. Oxford, Clarendon Press, 1990.

STRAWSON, Peter F. "Libertad y resentimiento", en su Libertad y resentimiento (trad. J. J. Acero). Buenos Aires, Paidós, 1996.

VILLA, Dana R. "Beyond Good and Evil: Arendt, Nietzsche, and the Aestheticization of Political Action", Political Theory, 20, 1992.

WallaCE, Jay. "Ressentiment, Value, and Self-Vindication: Making Sense of Nietzsche's Slave Revolt", en su Normativity and the Will. Oxford: Oxford University Press, 2006. aparecer), 2007.

. "The Argument of Resentment", Proceedings of the Aristotelian Society, 107 (por

Walzer, Michael. "Political Action: The Problem of Dirty Hands", Philosophy and Public Affairs, 2, 1973.

WiLliAmS, Bernard. "Nietzsche's minimalist moral psychology", en su Making sense of humanity. Cambridge: Cambridge University Press, 1995. 